\title{
La inclusión educativa en el aula regular: Un caso de síndrome de Asperger
}

\section{Educational Inclusion in the Regular Classroom: an Asperger Syndrome Case}

\author{
Fressy Andrade Ruiz ${ }^{1}$ \\ Universidad Estata a Distancia \\ San José, Costa Rica \\ fressyandrade@gmail.com
}

Recibido 05 de mayo de 2010 • Aceptado 31 de agosto de 2010

\begin{abstract}
Resumen.El siguiente artículo se generó a partir de la experiencia de la investigación final de la Maestría Formación de Formadores de Docentes de Educación Primaria, la cual se llevó a cabo por la División de Educación Rural del Centro de Investigación y Docencia en Educación (CIDE-UNA), en coordinación con la CECC (Coordinación Educativa y Cultural Centroamericana). La investigación es cualitativa con un enfoque interpretativo. El objetivo fundamental fue analizar el proceso de inclusión educativa en el aula regular de una persona con Síndrome de Asperger, entendido este como un tipo de discapacidad social. Se trabajó con el método de estudio de casos, dado que este permite la profundización en el estudio, con una niña de una escuela estatal de la zona urbana de San José-Costa Rica. Para obtener la información se emplearon tres técnicas: las entrevistas, los cuestionarios y documentación (expediente, registro de conductas, valoración psicológica) de la niña con Asperger. Se empleo la triangulación de fuentes como método de análisis. A partir del mismo se llegó a la conclusión de que en las escuelas se pueden atender niños (as) mal diagnosticados (as) con el Síndrome de Asperger; que en las escuelas del país aún se está lejos de lograr la inclusión educativa; pero que se están realizando esfuerzos por lograrlo. Para una intervención más oportuna, a partir del estudio se realizaron recomendaciones a la familia y a la escuela.
\end{abstract}

Palabras claves. Inclusión educativa, escuelas inclusivas, discapacidad social, Síndrome de Asperger.

Licenciada en Psicología. Universidad de Costa Rica. Bachiller en la Enseñanza de la Psicología (UCR). Magíster de la Maestría de Formación de Formadores de docentes de Educación Primaria. Profesora de la Escuela de Formación Docente (UCR) y supervisora de Práctica Docente de la Universidad Estatal a Distancia (UNED). 


\begin{abstract}
The following paper resulted from the final research project conducted for my Master's Degree in Teacher Training for Teachers of Primary Education $\left(1^{\text {st }}-6^{\text {th }}\right.$ grade of the Basic General Education). This research project was conducted under the supervision of the Rural Education Division of the Center for Research and Teaching in Education (CIDE-UNA, Spanish acronym), in coordination with the Central America Educational and Cultural Coordination (CECC). The research is qualitative with an interpretative approach. Our main objective was to analyze the process of inclusive education in the regular classroom for a person with Asperger's Syndrome, defined as a type of social impairment. The case study method was used in this research, as it allows a deeper study. A girl was chosen from a public school in an urban area of San José, Costa Rica. Three techniques were used to obtain information: interviews, questionnaires and documentation (personal file, behavior record, and psychological assessment) related to the girl with Asperger. The triangulation of sources was used as a method of analysis. The conclusion of the project was that regular schools may have children miss-diagnosed with Asperger's Syndrome, and that our schools are still far from achieving inclusive education, but efforts are being made to achieve it. For a more opportune intervention, some recommendations based on this study were provided to the family and the school of the girl with Asperger.
\end{abstract}

Keywords. Inclusive education, inclusive schools, social disability, Asperger’s Syndrome.

\title{
Introducción
}

La educación es un derecho y como tal los niños, niñas y adolescentes deben practicarlo en igualdad de condiciones. Por tal razón, desde hace aproximadamente dos décadas, se viene trabajando arduamente en el establecimiento de convenios y legislaciones que inviten a trabajar en la tarea de evitar la segregación y la discriminación en todas sus manifestaciones y que comprometan a los estados que aceptaron estos compromisos. Dentro de este marco se encuentra lucha contra la discriminación por discapacidad.

El paradigma de la educación inclusiva surge como respuesta a las limitaciones de la educación tradicional, así como a las insuficiencias resultantes de esta, por medio de la cual se evidenció una brecha entre la población infantil estimada como "normal" y otra considerada "especial". Dicha separación procedía, principalmente, de la incapacidad del sistema educativo para atender y respetar las particularidades educativas de sus estudiantes, más que de la imposibilidad de que ambas poblaciones compartieran el mismo centro educativo y las mismas oportunidades.

Por medio de la educación inclusiva se aboga por un sistema único en el que todo niño y niña reciba una educación acorde con sus necesidades educativas, la cual, en algunos casos, puede ser diferente.

Existen convenios y declaraciones internacionales como el Pacto Internacional de Derechos Económicos, Sociales y Culturales; la Convención sobre los Derechos del Niño; la Declaración de Salamanca; la Conferencia Mundial sobre Necesidades Educativas Especiales: Acceso y Calidad en 1994 y la Convención sobre los Derechos de las Personas con Discapacidad, más reciente, la cual establece la obligación de que los países aseguren inclusividad en el sistema educativo (Muñoz, 2007). Algunos de estos documentos se revisaron para la elaboración teórica de este artículo y de la investigación que le da origen.

Como parte de este paradigma de educación inclusiva, se ha logrado que muchos niños y niñas con condiciones personales y culturales diversas se encuentren en las aulas. Tal es el caso de la población con síndrome de Asperger (S. A.), población que en su mayoría logra funcionar cognitivamente en el aula regular; pero con evidente incapacidad para comprender el sentido e 
intencionalidad de la comunicación social, lo cual, según plantea la bibliografía consultada, debe entenderse como una discapacidad social.

Tomando como referencia que la inclusión educativa se fundamenta en el derecho de las personas a educarse por encima de las particularidades personales o culturales, y que la escuela es solo una parte de la sociedad y a su vez reflejo de esta, se tiene claridad de que este microsistema debe lograr formas cada vez más inclusivas de tratar las diferencias aceptándolas y atendiéndolas.

Es así como el problema de investigación que nos convoca se orienta a entender el proceso de inclusión educativa, en el aula regular de una escuela estatal costarricense, de una niña con síndrome de Asperger. Mismo que sustenta el objetivo general: analizar el proceso de inclusión educativa para determinar acciones concretas que se puedan introducir a la realidad del aula regular, a fin de que la persona con esta discapacidad pueda ser incluida con más éxito. Para abordarlo se trabajará con un estudio de caso.

\section{Aspectos teóricos}

\section{El paradigma de la inclusión en la educación}

Tradicionalmente las personas con algún tipo de discapacidad fueron atendidas en escuelas separadas o en clases especiales dentro de las escuelas ordinarias. No obstante, en los últimos años y a partir de la sensibilización y toma de conciencia de organismos internacionales como la UNESCO y la ONU, se ha movilizado a las naciones del mundo a realizar cambios graduales en materia de derechos humanos y derechos de la infancia en distintos ámbitos, entre ellos el educativo.

Es así como también se han realizado grandes esfuerzos por modificar y promover que los distintos grupos humanos tengan acceso real a otros servicios y, con ello, la oportunidad de una mejor calidad de vida.

Artavia (2005), haciendo un breve recuento de los distintos momentos por los que ha avanzado la Educación Especial en nuestro país, menciona que en Costa Rica, a partir de 1940, se crea una serie de servicios en las áreas de Educación Especial y Rehabilitación Física. Se promulgaron leyes, decretos y normas como instrumentos legales que servirían de apoyo a la rehabilitación y comienzan a funcionar las aulas recurso, como entes que brindan apoyo a la población estudiantil con limitaciones leves, dentro del sistema educativo.

Indica la misma autora que, a partir de la década de los años sesenta y setenta, comenzaron a tomar auge los conceptos de integración y normalización: “(...) la integración escolar procura incorporar a un sistema educativo normal a aquellos estudiantes que presentan algún tipo de retraso y problema de aprendizaje [para que] reciban el apoyo y la atención que requieran para alcanzar su desarrollo personal" (Artavia, 2005, p. 64). Con este enfoque se daba por sentado que las personas que se integraban eran diferentes a las "normales" y que se les otorgaba la oportunidad de integrarse a la comunidad de los no deficientes.

Sin duda, se seguía conceptualizando y llevando a la práctica hechos discriminatorios contra las personas y estudiantes que, con un distinto funcionamiento cognitivo, con alguna discapacidad física, sensorial o con diferencias culturales o étnicas, ingresaban a las escuelas. Se manejaba un parámetro de "normal" y otro de "deficientes". 
Siguiendo con el recuento histórico, se plantea que a partir de la década de los ochenta, en Costa Rica, se comienza a formar una mayor conciencia acerca de la atención y satisfacción de las necesidades de las personas con discapacidad, particularmente, en el contexto familiar y comunitario.

Para 1996, con la creación de la Ley 7600, conocida como "Ley de Igualdad de Oportunidades para la Persona con Discapacidad", se procura que, de manera obligatoria, las instituciones educativas públicas o privadas ofrezcan oportunidades para todos(as), con el fin de que se garantice una participación real y concreta en procesos educativos, y que procuren el desarrollo individual y social, en general.

Con esta Ley se gestiona, entre otras cosas, fomentar adecuaciones curriculares en el Sistema Educativo Costarricense, con el fin de mejorar la calidad de los aprendizajes, tomando en cuenta las diferencias que existan entre las personas y las regiones.

Aunque esto representaba un avance con el surgimiento del enfoque de la inclusividad, en esta misma década la diversidad pasa a ser valorada como elemento enriquecedor del proceso de aprendizaje y, en consecuencia, favorecedor del desarrollo humano, por lo que en el ámbito educativo debía ser respetada e incluida en todas sus manifestaciones.

S. Stainback y W. Stainback (2004) plantean que, por varias razones, se ha producido un cambio del concepto de integración por el de inclusión:

[Primero] porque comunica con mayor precisión y claridad lo que hace falta: hay que incluir a todos los niños [y las niñas] en la vida educativa y social de las escuelas y aulas de su barrio y no sólo colocarlos en clases normales. En segundo lugar, se está abandonando el término integración porque supone que el objetivo consiste en reintegrar a (...) algún grupo (...) que había sido excluido (...) En tercer lugar, el centro de atención de las escuelas inclusivas consiste en cómo construir un sistema que incluya y esté estructurado para satisfacer las necesidades de cada uno (...) (pp. 21-22).

Desde esta perspectiva, no se asume que las escuelas y aulas tradicionales sean suficientes ni que todos los alumnos tengan que ajustarse a lo que se ha diseñado para la mayoría. Por lo cual, se propone el enfoque de educación inclusiva para las escuelas regulares.

"La educación inclusiva implica que todos los niños y las niñas de una determinada comunidad aprendan juntos independientemente de sus condiciones personales, sociales o culturales, incluidos aquellos que presentan alguna condición especial que les dificulta el aprendizaje" (Delgado, 2007, p. 51).

En este sentido, la escuela se convierte en un espacio físico, social y emocional en el que la comunidad educativa colabora en conjunto con la institución, en la concreción de un proyecto educativo humanizante, que aspire a hacer realmente efectivos los derechos a la educación y a la igualdad de oportunidades. Según Ainscow (2001, p. 12), “(...) el objetivo consiste en reestructurar las escuelas en respuesta a las necesidades de los alumnos".

La escuela es un espacio óptimo para que los niños y las niñas sean acogidas sin discriminación. Este enfoque no es mejor para las personas con discapacidad o con problemas de aprendizaje, sino que es mejor para todos y todas, porque se basa en el respeto a la diversidad.

Dado que en las escuelas inclusivas, el aula es la unidad básica de atención, S. Stainback y W. Stainback (2004) recomiendan que las clases se organicen de forma heterogénea y se estimule e 
impulse a alumnos y maestros para que se apoyen mutuamente. Para ello, se deben ajustar aspectos relativos a la filosofía de clase, las reglas de aula, enseñanza adaptada al alumno (a), apoyo en el aula ordinaria, fomento de la comprensión de las diferencias individuales, flexibilidad; las cuales conforman sus características básicas.

\section{EI síndrome de Asperger: una discapacidad social en el aula regular}

Este síndrome fue descrito por primera vez por el pediatra austriaco Hans Asperger en el año 1944; sin embargo, su descubrimiento no se dio a conocer internacionalmente hasta la primera década de 1980, cuando la famosa psiquiatra británica, Lorna Wing publicó una revisión en lengua inglesa del trabajo pionero de Asperger (Martin, 2006).

Según la experta Martin, quien es especialista en el diagnóstico y tratamiento psicológico de los trastornos psiquiátricos y ha realizado importantes aportes al estudio de este cuadro clínico en el Reino Unido y España, este síndrome plantea una constelación de comportamientos agrupados en torno a tres núcleos de trastornos. A continuación se describen, tal como ella los presenta:

I. Trastorno cualitativo de la interacción social

II. Alteraciones de las habilidades pragmáticas del lenguaje y la comunicación social

III. Trastorno de la flexibilidad comportamental y mental

El primero, parte de que todos los niños con síndrome de Asperger presentan un conjunto de déficits significativos en las áreas del comportamiento social, la adquisición de las habilidades sociales, la comunicación no verbal y la reciprocidad emocional.

El segundo núcleo de trastornos está relacionado con la capacidad disminuida del individuo para la comunicación recíproca y empática. La tendencia a utilizar un lenguaje pedante, preciso, excesivamente formal y emocionalmente inexpresivo.

El tercer trastorno implicado se refiere a la rigidez comportamental e inflexibilidad cognitiva que el niño manifiesta en situaciones cotidianas. Exhiben un grado mayor o menor de severidad, un patrón restrictivo de conductas, intereses y actividades repetitivas y estereotipadas. (p. 54)

A pesar de las muchas investigaciones que se están llevando a cabo, no existen aún criterios uniformes sobre las posibles causas de este síndrome, pero la mayoría de hipótesis sugieren un origen genético y no se duda de su base orgánica.

De igual manera, es importante anotar que Hans Asperger originalmente creía que el trastorno no podía presentarse en niñas. Sin embargo, en la actualidad se sabe que las niñas también pueden estar afectadas por el S. A., si bien la prevalencia es “(...) significativamente inferior a la de los niños (2-3 niños: 1 niña)" (Gillberg, 1995, citado por Martin (2006, p. 19). 
Debido a que el estudio del caso se realizó con una niña, se procuró encontrar información concerniente a esta población; sin embargo, es sumamente escasa. Maristany (2002, “3.6.- Características del juego”, parr. 7) plantean lo siguiente:

Las niñas con síndrome de Asperger tienen características similares a las de los niños pero con expresión más suave. Son más propensas a hablar y menos a tener conductas disruptivas y agresivas como respuesta a emociones negativas o a la confusión. Son más pasivas, rechazan menos a sus compañeros y se dejan "cuidar" por ellos. Parece que las niñas aprenden con mayor facilidad por imitación conductas sociales, tono de voz y lenguaje corporal. Tienen tendencia a teatralizar. Son más propensas a crear "amigos imaginarios" y utilizan a las muñecas como sustitutivos de los amigos reales más allá de la edad en la que esta actividad se considera normal. Tienen intereses específicos, pero en general menos llamativos que los de los niños. Se apasionan con los animales, les gustan las poesías, almacenan información sobre ídolos del cine o de la música, etc. Conforme adquieren autonomía cuidan poco su aspecto externo y prefieren mantenerse niñas sintiendo cierto rechazo a los cambios de la pubertad.

Como se ha venido exponiendo en este apartado, aunque puede existir alguna particularidad por socialización y género, tanto las niñas como los niños con síndrome de Asperger tienen enormes desventajas para comprender y poder responder de forma espontánea en sus relaciones humanas, decrecidas por su rigidez en la comunicación.

"La gente con Asperger necesita asistencia en el desarrollo de sus habilidades sociales y comunicativas (...)" (Autism Society of America, 2004, Párr. 23), pues es una necesidad para estos niños y estas niñas lograr un nivel de funcionamiento que al menos les permita lograr pautas básicas de respuesta social, dado que el ser humano es eminentemente social, y como tal, persigue la interacción con sus iguales desde una etapa muy temprana de su desarrollo.

Baron-Cohen (citado por Martin, 2006, p. 61) menciona:

La mayoría de los niños con síndrome de Asperger se sienten desdichados en el colegio porque no son capaces de hacer amigos. Es difícil imaginarse lo que supone esta situación. La mayoría de nosotros damos por hecho que nos llevaremos suficientemente bien con la gente como para conseguir tener un grupo variado de amigos. Pero, tristemente, las personas con síndrome de Asperger suelen estar rodeadas de conocidos y extraños, pero no de amigos, en la forma en que nosotros entenderíamos ese concepto. Muchos de ellos son objeto de burlas porque no consiguen adaptarse, o no muestran ningún interés por ser incluidos. Su falta de conciencia social puede incluso resultar en que ni siquiera ellos traten de camuflar sus excentricidades. (...)

Desde esta perspectiva, se hace más sencillo visualizar los difíciles momentos, altamente sociales, por los que atraviesan las personas con síndrome de Asperger en los centros educativos. Existen otras particularidades del ser humano que pueden ser disimuladas, pero las de tipo social son muy visibles y la inhabilidad en su manejo muy censurada, especialmente por los iguales. 
A pesar de que no se conoce con exactitud su prevalencia en la población costarricense, estudios realizados en otros contextos parecen indicar que “(...) su incidencia es más alta que la del autismo o cualquier otro trastorno severo del desarrollo. Posiblemente se puedan dar 36 casos de síndrome de Asperger en una población de 10.000 en comparación con 5 casos en una muestra de la población de igual tamaño (...)" (Howlin, 1998, citado por Martin, 2006, p. 19).

En la práctica y en nuestro contexto, todos o casi todos los niños(as) con síndrome de Asperger asisten al aula regular. Dado que en su mayoría no presentan dificultades cognitivas, posiblemente son atendidos sin necesidad de recursos extraordinarios, a excepción de aquellos en quienes el síndrome se combina con un bajo perfil cognitivo o con un marcado déficit atencional. Por lo cual, su principal área de dificultad, que es la social, no es atendida.

No poder entenderse con los demás ha sido motivo de dificultades en el aula regular, en tanto provoca conflictos, peleas, burlas, entre otros; todo lo cual lleva a los docentes a intervenir como mediadores, censuradores, o a resolver maternalmente dificultades de interacción entre los escolares. Sin embargo, no visualizan que algunos de sus estudiantes recurrentemente tienen estos problemas porque sus habilidades sociales son muy básicas o porque, en algunos casos, pueden estar sufriendo síndrome de Asperger (S. A.). A lo que se suma que aunque los docentes y las docentes conozcan el diagnóstico médico, tampoco saben cómo proceder para entrenar a estos niños (as) para que logren mayor éxito social.

\section{Evaluaciones psicológicas: valoración psicométrica de la capacidad intelectual en el S. A.}

Según Martin (2006), la evaluación de la capacidad intelectual “(...) revela el estilo de aprendizaje particular de un niño facilitando la planificación de los programas educativos y estrategias de intervención (...)" y permite una “(...) estimación de su pronóstico acerca del futuro rendimiento académico (...)" (pp. 104-105).

En el caso que nos ocupa, la valoración de las competencias cognitivas se realizará por medio de la Escala de Inteligencia para Niños(as) Wechsler. La prueba indicada es el WISC-III, por grupo de edad, a partir del cual se podrá obtener un índice global de inteligencia e información suficiente para la elaboración de un perfil cognitivo individualizado. Se considera importante realizar esta prueba, ya que permite obtener información relevante para la escuela, a la vez que forma parte del diagnóstico diferencial del síndrome.

Martin (2006), dice que:

En la prueba es característico que el niño con síndrome de Asperger manifieste un avanzado desarrollo de las habilidades verbales (...) así como en las pruebas de memoria verbal y percepción auditiva (...) y a menudo una disfunción en el procesamiento de la información visoespacial que se manifiesta en la dificultad para la integración visomotora, percepción visoespacial, memoria visual y desarrollo de conceptos no verbales. (p. 105)

De ahí que es frecuente que presenten dificultades en el área de las matemáticas y de la escritura. Así como problemas en la combinación de las palabras, una gama restringida de significados y un déficit importante en la comprensión del lenguaje novedoso. Razón por la cual no utiliza el lenguaje de una manera socialmente apropiada. 
Los temas hasta acá abordados enmarcan aspectos teóricos que fundamentan el estudio de caso en mención y permiten realizar una valoración juiciosa del mismo, de modo que las recomendaciones sean pertinentes.

\section{Aspectos metodológicos}

La investigación se inscribe dentro del paradigma hermenéutico, de tipo exploratorio y crítico. El paradigma hermenéutico o interpretativo, dentro de la realidad educativa supone que el significado que las personas atribuyen a sus vivencias y las de otros, expresadas a través del lenguaje, son muy importantes dentro del proceso investigativo. Exploratorio, dado que en nuestro contexto no se registran investigaciones atinentes a la inclusión de esta población al sistema regular. Critico, porque la investigación se propone hacer evidente las necesidades de un grupo cuya discapacidad se invisibiliza en las aulas, pese a que existen leyes de inclusividad que los amparan, con lo cual quedan al descubierto las contradicciones del sistema. Por medio de las recomendaciones se ofrecen posibilidades de intervención que pueden ser incorporadas e implementadas en el sistema estatal de nuestro país.

Se trabajó con estudio de casos, que constituye un método de recolección de información de los más característicos del enfoque interpretativo. Las técnicas de recolección de información fueron la entrevista (a padres de familia, orientador, maestras de materias básicas y maestra de problemas de aprendizaje); el cuestionario a maestras(os) de tres materias especiales, un registro de conducta completado por la docente guía, algunas pruebas psicológicas (WISC-III y emocionales) y documentos oficiales de la institución (expediente).

Como criterios de selección para participar en el estudio, el niño o la niña participante debía tener un diagnóstico de síndrome de Asperger emitido por un especialista en Neurología; pertenecer a una escuela estatal y ser preferiblemente costarricense. Además, tanto la institución como los padres de familia deberían brindar las respectivas autorizaciones. En este caso, la persona que completó los criterios fue una niña de la escuela "La Isla de Moravia".

La información se analizó por medio de la técnica de triangulación de fuentes, los grandes temas son: la inclusión educativa, el síndrome de Asperger, y el caso propiamente dicho.

\section{Resultados de la investigación}

Por medio del análisis de la información, se organizó la misma en tres categorías. Estas son:

1. El entorno escolar y el proceso de inclusión educativa en el aula regular

2. Conocimiento que tienen los docentes sobre el síndrome de Asperger

3. El caso

De manera que los hallazgos de investigación más significativos fueron los siguientes: 


\section{El entorno escolar y el proceso de inclusión educativa en el aula regular}

Para los docentes entrevistados y los que contestaron el cuestionario, la educación inclusiva se respalda en la ley 7600. Cuatro de los siete docentes entrevistados identifican esta como la normativa vigente relacionada con el tema de inclusión educativa, dos no saben sobre leyes o convenios que la respaldan y uno dice conocer convenios de la ONU y UNESCO, pero no los recuerda.

La mayoría conceptualiza la inclusión como la oportunidad que tienen los niños y las niñas de compartir las aulas, sin discriminación. Esta idea tiene sustento teórico, no obstante prevalece la representación de que son los niños con necesidades educativas especiales (n.e.e) o bien con discapacidad los que se incorporan al sistema regular y allí reciben apoyos, principalmente, a través de adecuaciones curriculares y del aula recurso, la cual funciona en la escuela. No consideran las diferencias étnicas, sociales o económicas, como parte de la inclusividad.

Consideran que la escuela inclusiva es aquella que admite niños con n.e.e, "dándoles la oportunidad" de recibir un servicio educativo adecuado y, por medio de este, integrarse a la sociedad. Lo anterior hace suponer que están visualizando la inclusión como integración educativa.

El criterio teórico indica que, más que dar la oportunidad a estos niños, la inclusión se logra cuando toda la comunidad educativa percibe las diferencias como la norma y todos(as) se dan la oportunidad de compartir espacios, valorando la diversidad y preparando al centro educativo para atender las particularidades que cada uno y cada una tiene, no solo en cuanto al aprendizaje sino también en condiciones culturales, étnicas, de género, entre otras.

No obstante, la realidad es que, en la práctica, las escuelas atienden, principalmente, los casos de niños(as) con problemas de aprendizaje o trastornos emocionales, por lo cual es evidente que los docentes confundan estas condiciones con el síndrome en cuestión.

Parte de la preparación para respetar la diversidad, consiste en que la institución cuente con personal capacitado para la atención de estudiantes con necesidades educativas especiales, algún grado de discapacidad y la expresión de las diferencias humanas en sus distintas manifestaciones. El enfoque inclusivo propone que las personas no deben ser discriminadas y particularmente los que tienen discapacidades, aisladas, como ocurría en el pasado, sino que es la sociedad quien debe proveer los recursos para brindar la asistencia técnica y profesional a estos niños, niñas y jóvenes, en el aula.

A partir del análisis de la información se determina que el personal docente ubica el soporte a los estudiantes, primeramente, en el personal de apoyo (orientador, profesional en problemas de aprendizaje, profesional en trastornos de aprendizaje) y no en sí mismos. De hecho, remiten a estos niños y niñas al aula recurso para que allí se les ofrezca la ayuda individualizada, lo cual no es conveniente desde el enfoque inclusivo. De manera que, en aula, el aporte consiste en ejecutar algunas adecuaciones en aspectos de contenidos y evaluación, prioritariamente.

Dado que la colaboración del profesorado es fundamental dentro de la promoción de las escuelas inclusivas, se indagó si los docentes que participaron en el proceso educativo de la niña en estudio han recibido o reciben capacitación. Se encontró que ninguno ha recibido capacitación sobre el tema ni inducción para trabajar con esta niña, lo cual resulta preocupante puesto que estos (as) son incorporados (as) al aula regular y sus educadores no están preparados para atenderlos (las) adecuadamente. En su mayoría, tampoco buscan algún tipo de capacitación adicional. 
Como fortalezas de la escuela y de su personal, en función del proceso de inclusión de los estudiantes y particularmente del caso en estudio, se encontró que existe apertura y disposición por parte del equipo de apoyo, buena relación entre este y los docentes de aula regular y de materias especiales, así como una dirección volcada a facilitar a los docentes los procesos administrativos, cuya gestión favorece los cambios curriculares. De igual manera, se pudo constatar que se están realizando esfuerzos (rampas, ajuste de baños y puertas) para atender las particularidades de la población, según sea su condición y sus necesidades en el ámbito educativo y socio familiar.

Estas cualidades son positivas para establecer, con éxito, las bases de cooperación y coordinación entre el conjunto institucional, pues, tomando en cuenta la novedad del enfoque, es imprescindible la creatividad y la apertura de mente de los miembros de la escuela.

\section{Conocimiento que tienen los docentes sobre el síndrome de Asperger}

Respecto la segunda categoría, los hallazgos de la investigación fueron:

Se determinó, por medio del estudio de caso, que únicamente los profesionales de apoyo conocen sobre este síndrome, por la formación que han recibido. Lo cual representa una limitación para que la educación sea inclusiva y amplía la posibilidad de que, en la práctica, los docentes y las docentes de aula regular que atienden propiamente a la niña, muestren poca confianza al tratar el caso y le deleguen el trabajo al aula recurso, con lo cual se marca nuevamente la discriminación.

En cuanto al estilo de mediación pedagógica que utilizan con la niña en estudio, los docentes mencionan acciones que ellos toman, por ejemplo, estar vigilándola para que no se distraiga, integrarla con los compañeros, darle más explicaciones y reforzar los contenidos y la comunicación con el hogar. No obstante, estas son acciones muy básicas que se deben tomar con todos los estudiantes y no representan estrategias adaptativas de las prácticas áulicas propias para la problemática de la niña y la discapacidad social de fondo.

Se indagó respecto a nuevos aprendizajes y estrategias que los docentes han tenido que ir implementando a partir de las particularidades de la niña. Los docentes contestan: “(...) ayudarla a solucionar conflictos, mediar con ella, con los compañeros, la paciencia, el refuerzo positivo". Un docente menciona que no ha tenido que hacer nada diferente. Al igual, estas son las acciones frecuentes que un educador debe implementar en el aula ante situaciones esperadas, por lo que se considera que no se han implementado estrategias particulares con la niña.

Desde este punto de vista, la situación nos lleva a considerar que los docentes no pueden realizar la inclusión porque no saben cómo ejecutarla o la conceptualizan únicamente como "adecuaciones curriculares", porque desconocen las particularidades del síndrome de Asperger y de los estudiantes que lo portan.

También se debe considerar que esto se puede deber a que la estudiante en cuestión no se encuentre bien diagnosticada, por lo que no amerite una intervención distinta a la que se ha efectuado hasta el momento: adecuaciones por problemas de aprendizaje, contención emocional y apoyo en la resolución de conflictos. 


\section{El caso}

Sobre la tercera categoría los hallazgos de la investigación fueron:

El diagnóstico por síndrome de Asperger de esta niña fue efectuado por un especialista en Neurología. Previamente había sido valorada en una Clínica de la Caja Costarricense de Seguro Social, en la cual se le diagnosticó un trastorno por Déficit Atencional.

Por los reportes que ofrecen los docentes entrevistados y la familia, se concluye que esta condición de déficit atencional se mantiene, con comportamientos típico: la desatención, la impulsividad y la pérdida de la concentración conforme avanza el día. Los docentes informan que tiene mayor concentración durante la mañana, unos 40 minutos, dependiendo de la actividad, ya que cuando se trata de ejercicios de aplicación en actividades que le gustan o domina logra más tiempo.

Sin embargo, por medio de la documentación de la niña (la valoración psicológica, el análisis del expediente y registros de conducta), las entrevistas y la revisión de cuestionarios efectuados al cuerpo docente; instrumentos de recolección de información a través de los cuales se indagó sobre los núcleos de trastornos propios del Asperger y aspectos del desarrollo evolutivo, se determina que no cumple con varios de los criterios diagnósticos del S. A. Entre los cuales destacan:

En lo intelectual: en la prueba de Wechsler, WISC-III, la niña obtiene una puntuación global de 65. El establecimiento diagnóstico del síndrome de Asperger requiere, de acuerdo con los sistemas oficiales de la DSM y ICD, que el niño satisfaga el criterio diagnóstico de "ausencia de retraso cognoscitivo" (superior a 70), el cual debe ser obtenido a través de la administración de tests cognitivos psicométricos. La puntuación inferior indica que existen dificultades de tipo intelectual que pueden estar justificando algunos de sus comportamientos (razonamiento, conducta social y emocional) y no necesariamente Asperger.

Lo anterior también permite comprender problemas de aprendizaje detectados a lo largo de su vida escolar y cierto deterioro que los docentes han notado. Las dificultades aparecen tanto en las áreas perceptuales como en las verbales.

Las limitaciones en el área visoespacial son significativas, así como en su capacidad viso motora y explican, de alguna manera, la disgrafía y las inversiones persistentes en las letras b y d, lo cual no se presenta al hablar o leer. Si se toma en cuenta que, además, presenta un problema visual y que la niña se niega a usar lentes, las omisiones son aún más probables. Se debe prestar un especial interés a la dificultad que muestra en las distintas dimensiones de la memoria, lo cual perjudica su rendimiento generalizado. Estos problemas de aprendizaje no son exclusivos del síndrome de Asperger, tampoco suelen ser comunes.

En la conducta social: muestra avances significativos (no típicos de las personas con Asperger), entre estos: infiere sobre los ejemplos de la vida cotidiana y saca conclusiones que logra aplicar a su vida, manifiesta interés por estar con sus congéneres y hace esfuerzos fructíferos por integrarse, ha tenido cambios progresivos en la comunicación con sus docentes, madre e iguales; se muestra afectuosa y responde a los estímulos afectivos de su ambiente. No muestra rigidez en los patrones de comunicación y la falta de comprensión que indican las maestras puede deberse más a su pensamiento concreto y limitaciones de tipo intelectual ya señaladas.

Es poco expresiva verbal y gestualmente, pero logra establecer contactos afectivos y representar, con gestos, sus palabras o emociones. Asimismo, comprende los significados sociales 
de los gestos y responde a ellos. Lo que le cuesta comprender más es el significado de algunas palabras; sin embargo, muestra poca riqueza léxica. Su entonación no es monótona ni rígida. Logra descifrar el significado oculto de consejos que su madre le da. Utiliza la sonrisa social, logra el contacto ocular de manera apropiada. Es una niña que se muestra tímida e insegura, lo cual también influye en que se retraiga y evite el contacto social; no obstante, si logra el contacto, no rehúye. Ha logrado incorporarse a un grupo de iglesia y con sus primos participa en juegos organizados.

En lo emocional: según la valoración psicológica, muestra indicadores de inmadurez emocional: inseguridad, retraimiento, oposicionismo, capacidad en el manejo del enojo y frustración por debajo de la edad y son el llanto y el enojo las principales fuentes de respuesta.

A pesar de no ser una niña expresiva emocionalmente, la madre y las educadoras coinciden en que es una niña sensible y cariñosa, que busca demostrar su afecto por medio de abrazos y, últimamente, con palabras afectuosas, pese a que con mamá se muestra desobediente y resentida, si esta no le dedica el tiempo suficiente.

Para las maestras es notoria la necesidad que muestra por ser reconocida en su trabajo y cambios. Le gusta exhibir lo que hace bien y que le reconozcan su esfuerzo, si está linda y demás. Muestras gestos de alegría y orgullo cuando esto ocurre. Responde al estímulo positivo de los docentes y con estas es obediente y respetuosa.

Ha sido egoísta y poco empática ante las necesidades de otros(iguales), sin embargo, en la actualidad, se ha notado más involucrada y proactiva con lo que ocurre a su alrededor y que demanda una respuesta emotiva apropiada, por ejemplo, en un accidente que la abuela sufrió en la casa, ella fue la que resolvió.

En el último año (2009), su conducta ha sido la de una niña más controlada en la expresión de sus emociones negativas, con lo cual la falta de control del enojo, frecuente en la infancia, ha disminuido notoriamente. Manifiesta más autonomía tanto en sus deberes escolares como familiares, muestra más compromiso con el cambio hacia conductas prosociales.

En el desarrollo motor: por otro lado, es una niña que muestra habilidades motoras, principalmente en la motora fina, en las materias de artes es donde más destaca. Esto tampoco es frecuente en niños(s) con S. A. En la motora gruesa si muestra dificultades, no limitantes, para la ejecución de actividad física.

Todos estos aspectos, así como el avance positivo progresivo en los distintos núcleos de trastornos asociados con este síndrome, llevan a considerar que el diagnóstico debe esclarecerse, así como brindarle un seguimiento clínico y académico oportuno, ya que persisten las condiciones de déficit atencional y los problemas de aprendizaje, pero se duda de la presencia de síndrome de Asperger.

\section{Discusión}

En nuestro país se han aceptado convenios internacionales que privilegian el establecimiento de un estado de derecho, en el cual las personas sean tratadas sin discriminación y se les facilite el acceso a las oportunidades, principalmente cuando se trata de las personas menores de edad.

Debido a que las condiciones intelectuales, sociales, económicas, étnicas, entre otras, son propias para cada individuo y en la escuela es donde más se hacen evidentes, convendría capacitar de 
una forma especial a al personal docente del aula regular, para que discrimine ciertas problemáticas y pueda atender, de forma inclusiva, a los niños y niñas. Para ello será necesario, también, realizar cambios en la forma como se organizan las escuelas, el tamaño de los grupos y otros aspectos, pues estas condiciones impiden, en ocasiones, que un docente con buena formación y disposición logre realizar, óptimamente, su trabajo.

El síndrome de Asperger es una de los muchas condiciones que una persona puede tener, determina particularidades en la manera de comprender e insertarse en el mundo; no obstante, su prevalencia es relativamente pequeña y muy disimulada (en lo cognitivo), comparada con la de otros padecimientos, pero no menos significativa, en cuanto a los trastornos que provoca en el aula y particularmente al niño o niña que lo sufre, al no poder comprender el mundo social en que vive. Ante esto, una escuela que haya comprendido el enfoque de la inclusividad y trabaje con base en este, se convierte en la opción que se posee para ir construyendo la tan deseada sociedad justa y con equidad.

\section{Conclusiones}

- La escuela participante en la investigación no es una escuela inclusiva; sin embargo, cuenta con los recursos técnicos, el personal e instalaciones que le posibilitan funcionar como una escuela cada vez más inclusiva. De hecho, en el caso de la niña en estudio, la escuela asumió, con la mejor actitud, por medio del equipo técnico, las adecuaciones y otros apoyos que la niña ha ido requiriendo, a fin de atender sus necesidades académicas y sociales. El proceso no se logra de una manera efectiva, debido al desconocimiento del personal de aula regular sobre cómo trabajar con niños con ciertas particularidades y a que el personal de apoyo no les ha brindado la respectiva inducción.

- Se determina que a nivel institucional se sigue manejando el enfoque de integración educativa, el cual es entendido por muchos como inclusión. Por lo cual, se aborda la atención del estudiante con apoyos externos al aula (aula recurso). En el aula propiamente, por medio de las adecuaciones curriculares y sin involucrar a los otros estudiantes dentro de un ambiente colaborativo.

- Se estima que el desarrollo evolutivo de la niña muestra comportamientos predominantemente en el área social y en el desarrollo motor, asociados con el síndrome de Asperger, por lo cual el diagnóstico puede resultar confuso. Sin embargo, al analizar la información procedente de distintas fuentes se concluye que no muestra presencia contundente de síntomas en los núcleos de trastornos asociados con este síndrome. El déficit cognitivo podría explicar muchas de las características sociales, motoras y emocionales que presenta, las cuales son compartidas con otras categorías diagnósticas. Por lo cual, se debería clarificar el diagnóstico, así como darle seguimiento a nivel profesional para su adecuado tratamiento, en conjunto con los especialistas de la escuela.

- Debe considerarse que la niña fue evaluada por el neurólogo en el 2006 y por la Clínica de la CCSS en el 2005, lo cual llama la atención a padres y madres de familia respecto a no descuidar el tratamiento que dan a sus hijos(as) cuando se diagnostica algún padecimiento de este tipo. El trastorno de déficit atencional se mantiene, sin embargo, es dudosa la presencia de un síndrome de Asperger. 
- Tratándose de que el enfoque actual propuesto por convenios internacionales y acogido por el Ministerio de Educación Pública de nuestro país es el de la inclusión educativa, el cual es mejor para todos y todas, ya que se basa en el respeto a la diversidad y en la ayuda mutua, es importante continuar haciendo esfuerzos para que el personal y toda la comunidad educativa avancen hacia la reestructuración y la escuela inclusiva.

- $\quad$ Este estudio generó reflexiones que permiten hacer algunas recomendaciones a la escuela estatal participante, entre estas: continuar haciendo esfuerzos para que el personal y toda la comunidad educativa avancen hacia la reestructuración y la escuela inclusiva, para lo cual es necesario capacitarles o bien que cada uno se muestre interesado por su autoformación y busque estrategias de actualización en estos temas. Fomentar los vínculos, el compromiso, la creatividad, el trabajo cooperativo y la apertura de mente, entre el personal. Que la asistencia y el apoyo prestados a los alumnos para ayudarles a conseguir los objetivos curriculares se puedan brindar en su espacio natural de aula y no en el aula recurso, la cual marca diferencias. Fomentar las redes naturales de apoyo entre los estudiantes, como la tutoría, círculos de conversación, aprendizaje colaborativo, y todas aquellas actividades que fomenten las habilidades sociales, el juicio y desarrollo moral. Que los estudiantes aprendan a valorar y aprovechar las diferencias individuales de manera que estas no se conviertan en motivo de popularidad ni marginación, y resulten nocivas para la formación del autoconcepto de estudiantado. En el caso de que la escuela cuente con docentes de apoyo, estos deben brindar la inducción a los docentes de grupo regular, cuando tengan que atender a niños con alguna discapacidad o particularidad, esto debido a que el seguimiento que brinda el docente en el aula es fundamental dentro del enfoque de inclusividad.

- También se pueden realizar algunas recomendaciones para el Ministerio de Educación, ente rector de la educación estatal en nuestro país. Entre ellas: continuar formando al personal docente en materia de inclusión educativa, particularmente a los del aula regular, quienes atienden a los niños(as) en su contexto, desde el enfoque de la inclusividad. Esto implica capacitación respecto a estrategias para lograr la inclusividad, manejo de grupo para un ambiente colaborativo y respetuoso de las diferencias, manejo de discapacidades sensoriales, sociales, diversidad cultural y étnica, etc.

- Es importante que se tome en cuenta que si el Ministerio de Educación abrazó este paradigma, debe procuran brindar a los educadores condiciones para que puedan plasmarlo en la realidad, por lo cual se deben considerar aspectos de preparación, pero también los que conciernen a la parte administrativa: el tamaño de los grupos, materiales, asesoría técnica.

- $\quad$ No debe quedar por fuera que el docente requiere tener, ante todo, una buena actitud y valorar este enfoque, no como uno más, sino como el que realmente abre la posibilidad de generar una sociedad justa y una cultura de paz, propósito que todo educador debe contemplar dentro de su misión social.

\section{Referencias bibliográficas}

Ainscow, M. (2001). Desarrollo de escuelas inclusivas. Ideas propuestas y experiencias para mejorar las instituciones escolares. Madrid: Narcea de Ediciones. 
Artavia, J. (2005). Actitudes de las docentes hacia el apoyo académico que requieren los estudiantes con necesidades educativas específicas. Revista Pensamiento Actual, 5(6), 61-70.

Autism Society of America. (2004). El síndrome de Asperger. Recuperado de http://www.ct-asrc. org/PDF/spanish2.pdf

Delgado, W. (2007). Inclusión: principio de calidad educativa desde la perspectiva del desarrollo humano. Revista Educación, 31(2), 45-58.

Maristany, M. (2002). El alumno con síndrome de Asperger en la escuela primaria. Asociación Asérger Argentina. Recuperado de http://www.asperger.org.ar/index.php?option=com content \&view $=$ article $\&$ id $=60$

Martin, P. (2006). El síndrome de Asperger ¿Excentricidad o discapacidad social? Madrid: Alianza Editorial.

Muñoz. V. [Relator principal ]. (2007). El derecho a la educación de las personas con discapacidades (Informe A/HRC/4/29). Recuperado de www.conadis.salud.gob.mx/descargas/ pdf/07VernorMunozONU.pdf

Stainback, S. y Stainback, W. (2004). Aulas inclusivas. Un nuevo modo de enfocar y vivir el currículo. Madrid: Narcea de Ediciones. Recuperado de http://books.google.com/books?id=36TU

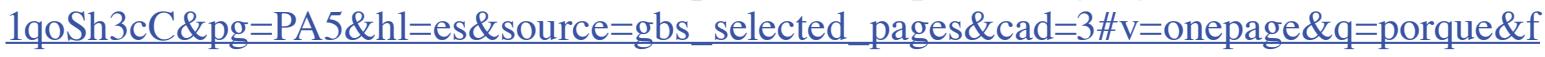
=false 